\title{
Effects of exenatide and liraglutide on 24-hour glucose fluctuations in type 2 diabetes
}

\author{
Jo Nagakura ${ }^{1)}$, Tadashi Yamakawa ${ }^{1)}$, Masataka Taguri ${ }^{2)}$, Hirohisa Tsuchiya ${ }^{3)}$, Erina Shigematsu ${ }^{1)}$, \\ Jun Suzuki ${ }^{1)}$, Satoshi Morita ${ }^{4)}$, Kazuaki Kadonosono ${ }^{5)}$ and Yasuo Terauchi ${ }^{6)}$ \\ 1) Department of Endocrinology and Diabetes, Yokohama City University Medical Center, Yokohama, Japan \\ 2) Department of Biostatistics and Epidemiology, Yokohama City University Medical Center, Yokohama, Japan \\ 3) Department of Diabetes, Yokosuka City Hospital, Yokosuka, Japan \\ 4) Department of Biomedical Statistics and Bioinformatics, Kyoto University Graduate School of Medicine, Kyoto, Japan \\ 5) Department of Ophthalmology, Yokohama City University Medical Center, Yokohama, Japan \\ 6) Department of Endocrinology and Metabolism, Yokohama City University School of Medicine, Yokohama, Japan
}

\begin{abstract}
We evaluated the influence of short-term treatment with exenatide twice daily or liraglutide once daily on daily blood glucose fluctuations in 40 patients with type 2 diabetes inadequately controlled by sulfonylureas. The patients in a multicenter, open-label trial were randomly assigned to receive add-on exenatide $(10 \mu \mathrm{g} / \mathrm{day}, \mathrm{n}=21)$ or add-on liraglutide (0.3-0.9 mg/day, $\mathrm{n}=19)$, and underwent 24-hour continuous subcutaneous glucose monitoring. There was no significant between-group difference in glucose fluctuations during the day, as assessed by calculating mean amplitude of glycemic excursion (MAGE) and standard deviation (SD). However, the mean blood glucose levels at 3 hours after breakfast and dinner were significantly lower in the exenatide group than the liraglutide group (breakfast: $127.3 \pm 24.1 \mathrm{vs.} 153.4 \pm 28.7$ $\mathrm{mg} / \mathrm{dL} ; p=0.006$, dinner: $108.7 \pm 17.3 v \mathrm{~s} .141 .9 \pm 24.2 \mathrm{mg} / \mathrm{dL} ; p<0.001)$. In contrast, mean blood glucose levels and their $\mathrm{SD}$ were significantly lower between $0000 \mathrm{~h}$ and $0600 \mathrm{~h}$ in the liraglutide group than the exenatide group (average glucose: $126.9 \pm 27.1 v s .107 .1 \pm 24.0 \mathrm{mg} / \mathrm{dL} ; p=0.029$, SD: $15.2 \pm 10.5 v s .8 .7 \pm 3.8 ; p=0.020$ ). Both groups had similar glucose fluctuations despite differences in 24-hour blood glucose profiles. Therefore, each of these agents may have advantages or disadvantages and should be selected according to the blood glucose profile of the patient.
\end{abstract}

Key words: Exenatide, Liraglutide, Randomized trial, Type 2 diabetes, Continuous subcutaneous glucose monitoring

THERE is increasing evidence that glycemic abnormalities such as rapid intra-day fluctuations of glucose might play an important role in the development of complications of diabetes [1]. Both cross-sectional and longitudinal studies have demonstrated that marked glycemic variability, including acute postprandial glucose excursions, increases the risk of diabetic complications [2-7]. Most of these investigations of glucose variability have been based on conventional statistical parameters, such as the standard deviation, coefficient of variation, postprandial peak, or area under the concentration $v s$. time curve [8].

Glucagon-like peptide-1 (GLP-1) enhances insulin

Submitted Aug. 21, 2015; Accepted Nov. 25, 2015 as EJ15-0500 Released online in J-STAGE as advance publication Jan. 5, 2016 Correspondence to: Tadashi Yamakawa, M.D., Ph.D., Department of Endocrinology and Diabetes, Yokohama City University Medical Center, 4-57 Urafunecho, Minami-ku, Yokohama 232-0024, Japan. E-mail: yamakat@urahp.yokohama-cu.ac.jp secretion and inhibits glucagon release to reduce postprandial hyperglycemia, and it may also suppress acute glucose fluctuations [9]. Exendin-4, originally isolated from the saliva of the Gila monster, shares 53\% sequence homology with the initial 30 amino acids of human GLP-1 [10]. Synthetic exendin-4 (exenatide) is resistant to cleavage by dipeptidyl peptidase 4 and has a half-life of approximately 3.5-4 hours after subcutaneous injection in humans, with its peak action being noted at 2-3 hours after injection [11, 12]. Treatment with exenatide achieves a better postprandial glucose profile than once-daily insulin glargine [13], probably due to the pleiotropic effects of the former drug. Exenatide shares several glucose-lowering actions with the naturally occurring incretin hormone GLP-1, including the promotion of glucose-dependent insulin secretion, inhibition of inappropriately high glucagon secretion, slowing of gastric emptying, and reduction of food intake [11, 14-18]. 
Liraglutide is a human GLP-1 analog that is effective for improving glycemic control when administered once daily. It is based on the structure of native GLP-1, with an amino acid substitution (lysine for arginine at position 34) and attachment of a C16 acyl chain via a glutamoyl spacer to lysine at position 26 [19]. Liraglutide is absorbed slowly, with the maximum serum concentration being reached at 10-14 hours after administration, and it has a half-life of 11-13 hours [19].

Minimally invasive continuous glucose monitoring (CGM) can be employed to detect intra-day glucose fluctuations that are impossible to identify with a standard blood glucose meter [20]. Exenatide was reported to improve glucose variability assessed by CGM [21], while liraglutide was found to narrow the range of circadian glucose variation in Japanese patients with type 2 diabetes [22]. However, direct comparison of the effects of these 2 drugs on 24-hour glucose fluctuations has not yet been performed in Japanese patients. Therefore, the present study was performed using CGM to examine the effects of liraglutide $(0.9 \mathrm{mg}$ once daily) and exenatide ( $5 \mu \mathrm{g}$ twice daily) on blood glucose fluctuations in Japanese patients with type 2 diabetes inadequately controlled by sulfonylurea therapy.

\section{Materials and Methods}

\section{Patients}

Patients with type 2 diabetes attending Yokohama City University Medical Center and Yokosuka City Hospital were enrolled in this study. The inclusion criteria were men or women between the ages of 20 to 85 years who showed inadequate glycemic control (hemoglobin A1c [HbA1c]: $6.9-16.6 \%$ ) with sulfonylurea therapy. The following patients were excluded: (1) patients with a history of diabetic ketoacidosis or diabetic coma within 6 months prior to study entry, (2) patients with a history of cardiac failure, (3) patients scheduled to undergo surgery during the observation period of this study, (4) patients with severe infection or severe trauma, (5) female patients who were pregnant or breast-feeding, (6) patients with renal insufficiency (serum creatinine $>132.6 \mu \mathrm{mol} / \mathrm{L}$ or estimated glomerular filtration rate (e-GFR) $<30 \mathrm{~mL} / \mathrm{min}$ ), (7) patients with severe liver dysfunction, (8) patients on insulin therapy, (9) patients with a history of a hypersensitivity to exenatide or liraglutide, and (10) other patients who were judged to be inappropriate for the study by the attending physician. The study protocol complied with the Declaration of Helsinki and was approved by the Ethics Committees of Yokohama City University School of Medicine (Yokohama, Japan) and its affiliated hospitals. All patients provided written informed consent.

\section{Study protocol}

This was an open-label, randomized controlled trial conducted at two study centers in Japan. Recruitment and follow-up were performed from December 2011 to April 2014 according to the above-mentioned criteria. The protocol of this study was reviewed and approved by the Institutional Review Boards of Yokohama City University Medical Center and Yokosuka City Hospital, and the study was registered with the University Hospital Medical Information Network registry (UMIN 000006799). Forty subjects were hospitalized and randomly assigned at a 1:1 ratio to the exenatide group $(10 \mu \mathrm{g} / \mathrm{day})$ or the liraglutide group ( $0.3-0.9$ /day) by the permuted block method using central computer-based randomization.

Three adjustment factors were used for allocation, which were $\mathrm{HbA} 1 \mathrm{c}(\mathrm{HbA} 1 \mathrm{c}<9 \%$ or $\geq 9 \%)$, age $(<60$ years old, $\geq 60$ and $<75$ years old, or $\geq 75$ years old), and sex. The allocation order per block was selected randomly from all hypothetical combinations. Since the block size was 4 , the order of patients in a block was selected randomly from six combinations (AABB, $\mathrm{ABAB}, \mathrm{ABBA}, \mathrm{BAAB}, \mathrm{BABA}$, and BBAA).

Exenatide was administered twice daily, before morning and evening meals, while liraglutide was given once daily before breakfast. The mean daily dose of exenatide was $10 \mu \mathrm{g}$ ( $5 \mu \mathrm{g}$ twice a day) in all patients. Liraglutide was escalated at 2-day intervals from the initial dose of $0.3 \mathrm{mg} /$ day to $0.6 \mathrm{mg} /$ day and then to $0.9 \mathrm{mg} /$ day. However, the dose of liraglutide was not increased further if the patient had a blood glucose level $\leq 90 \mathrm{mg} / \mathrm{dL}$ during the study period. As a result, the final daily dose of liraglutide was $0.9 \mathrm{mg}$ in 11 patients, 0.6 $\mathrm{mg}$ in 5 patients, and $0.3 \mathrm{mg}$ in 1 patient, with the mean dose of liraglutide being $0.8 \pm 0.2 \mathrm{mg} /$ day. A sulfonylurea was administered concomitantly in both groups. Glimepiride was given to all patients in the exenatide group at a mean dose of $1.0 \mathrm{mg} /$ day $(0.5-3 \mathrm{mg})$, and 16 patients from the liraglutide group also received glimepiride at a mean dose of $0.9 \mathrm{mg} /$ day $(0.5-3 \mathrm{mg})$. One patient from the liraglutide group received glibenclamide at a dose of $2.5 \mathrm{mg}$ /day. All patients ate standard hospital meals that had a daily energy content of 
$25 \mathrm{kcal} / \mathrm{kg}$ of ideal body weight. After 6 days of treatment, blood glucose levels were measured for three consecutive days by CGM (CGMS-gold and i-Pro2; Medtronic Minimed, Northridge, CA, USA). The following parameters were calculated by using CGM data obtained on the second day of monitoring: (1) the mean amplitude of glycemic excursion (MAGE), (2) 24-hour mean blood glucose level and SD, (3) fasting blood glucose level, (4) mean blood glucose level at 3 hours after each meal, (5) mean blood glucose level and standard deviation (SD) between $0000 \mathrm{~h}$ and $0600 \mathrm{~h}$, and (6) percentage of time with hypoglycemia $(<70 \mathrm{mg} / \mathrm{dL})$ or hyperglycemia ( $>180 \mathrm{mg} / \mathrm{dL})$. In addition, a 6-point (before and $120 \mathrm{~min}$ after each meal) self-monitored blood glucose (SMBG) profile was obtained at baseline and on day 7 using a blood glucose meter (Glutest Every; Sanwa Kagaku Kenkyusho, Aichi, Japan).

The plasma level of HbA1c, C-peptide immunoreactivity (CPR) index, and 24-hour urinary CPR level were also determined. The subjects all ate identical meals during hospitalization and were advised not to change their level of exercise.

\section{Statistical analysis}

No study has yet investigated whether liraglutide or exenatide is better for inhibiting blood glucose variability. Accordingly, calculation of the sample size for this study was performed carefully in order to predict intergroup differences at a certain level of accuracy. Based on the results of previous studies in which CGM was performed after administration of liraglutide and exenatide $[21,22]$, the SD of the primary efficacy parameter was calculated to be $15 \mathrm{mg} / \mathrm{dL}$. Using this assumption, the relationship between the overall width of the $95 \%$ confidence interval and the size of each group was calculated, revealing that the confidence interval was less than $10 \mathrm{mg} / \mathrm{dL}$ with 18 patients per group. If the delta of the mean SD of the difference between the two groups is assumed to be approximately $5 \mathrm{mg} / \mathrm{dL}$, statistical significance could be demonstrated with this group size. Therefore, assuming that some unevaluable patients might be enrolled, the sample size was set as 20 patients per group for a total of 40 patients.

Results are presented as the mean and standard deviation or as frequencies. The unpaired $t$-test was used to compare values between patients taking different drugs, with the level of significance being set at $p<$ 0.05 . Analyses were performed using SPSS version 22 (SPSS Inc. Chicago, IL, USA).

\section{Results}

Forty patients were randomized, and 35 patients completed the study and were included in this analysis. Three of the 21 patients in the exenatide group dropped out before completion of the study, as did 2 of the 19 patients in the liraglutide group. Of these 5 dropouts, 2 were due to withdrawal of consent, 1 was caused by a change of medication, and 2 were because CGM could not be performed (Fig. 1). Adverse reactions that occurred during the study period included mild gastrointestinal symptoms such as anorexia and nausea in both groups. These symptoms occurred in 6 patients from the exenatide group and 3 patients from the liraglutide group, but it was possible to continue treatment in all patients. There were no serious adverse reactions.

There were no significant between-group differences of baseline patient characteristics, including the age, sex, body mass index, duration of diabetes, HbAlc, CPR index, urinary CPR, and renal function (Table 1).

CGM was performed 7 days after the initiation of treatment and the results are shown in Fig. 2. There were no significant differences between the two groups with respect to the 24-hour mean blood glucose level, $\mathrm{SD}$, and MAGE. There were also no significant differences of early morning fasting blood glucose levels.

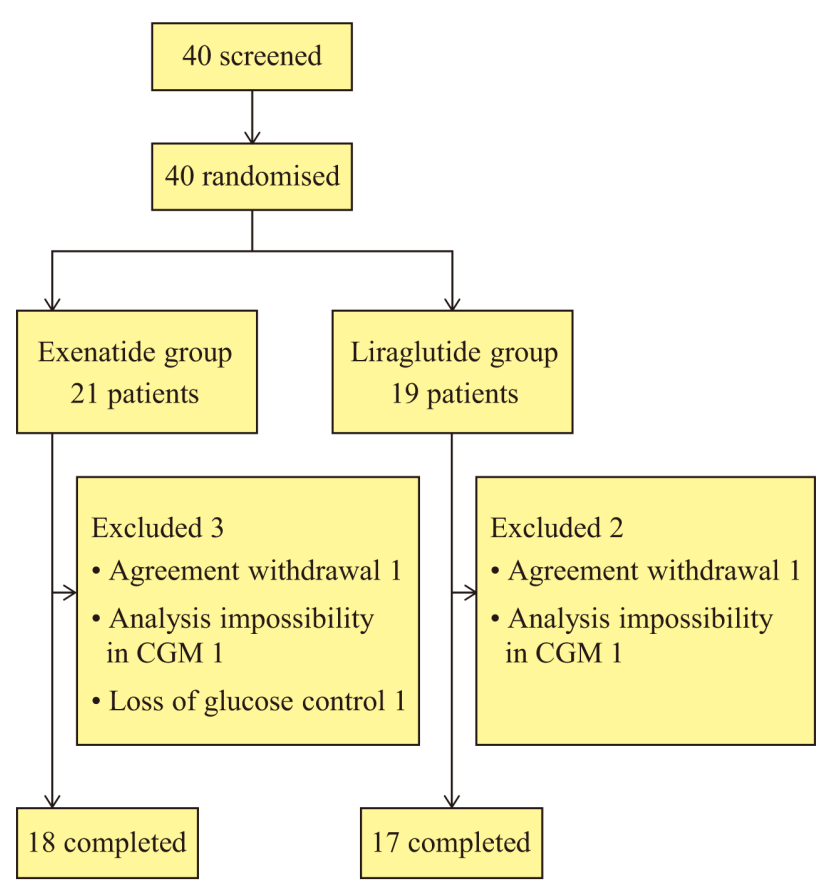

Fig. 1 Assessment, randomization, and follow-up of the study patients. 
However, there were differences in the changes of blood glucose after breakfast and dinner, as well as differences of the glucose fluctuations between nighttime and early morning. The mean blood glucose level at 3 hours after breakfast was $127.3 \pm 24.1 \mathrm{mg} / \mathrm{dL}$ in the exenatide group and $153.4 \pm 28.7 \mathrm{mg} / \mathrm{dL}$ in the liraglu- tide group, being significantly lower in the former group $(p=0.006)$. Similarly, the mean blood glucose level at 3 hours after dinner was $108.7 \pm 17.3 \mathrm{mg} / \mathrm{dL}$ in the exenatide group and $141.9 \pm 24.2 \mathrm{mg} / \mathrm{dL}$ in the liraglutide group, also being significantly lower in the former group $(p<0.001)$. However, mean blood glucose level

Table 1 Clinical and biochemical characteristics of patients

\begin{tabular}{|c|c|c|c|}
\hline & $\begin{array}{l}\text { Exenatide } \\
(\mathrm{N}=18)\end{array}$ & $\begin{array}{l}\text { Liraglutide } \\
(\mathrm{N}=17)\end{array}$ & $p$ value \\
\hline Age (years) & $61.1 \pm 10.3$ & $61.9 \pm 9.9$ & 0.809 \\
\hline Sex (male/female) & $12 / 6$ & $11 / 6$ & 0.903 \\
\hline BMI $\left(\mathrm{kg} / \mathrm{m}^{2}\right)$ & $27.7 \pm 5.5$ & $25.3 \pm 3.4$ & 0.137 \\
\hline Disease durations (years) & $6.2 \pm 6.2$ & $10.5 \pm 8.6$ & 0.097 \\
\hline HbAlc $(\%)$ & $9.6 \pm 1.3$ & $10.1 \pm 2.0$ & 0.457 \\
\hline Fasting plasma glucose $(\mathrm{mg} / \mathrm{dL})$ & $152.6 \pm 22.0$ & $171.1 \pm 38.1$ & 0.084 \\
\hline Doses of glimepiride (mg/day) & $1.0 \pm 0.7$ & $0.9 \pm 0.6$ & 0.601 \\
\hline AMY (IU/L) & $64.7 \pm 21.7$ & $78.4 \pm 43.7$ & 0.260 \\
\hline $\mathrm{eGFR}\left(\mathrm{mL} / \mathrm{min} / 1.73 \mathrm{~m}^{2}\right)$ & $77.3 \pm 9.6$ & $82.1 \pm 19.2$ & 0.358 \\
\hline $\mathrm{TC}(\mathrm{mg} / \mathrm{dL})$ & $179.0 \pm 28.3$ & $199.6 \pm 36.7$ & 0.078 \\
\hline $\mathrm{TG}(\mathrm{mg} / \mathrm{dL})$ & $135.8 \pm 66.2$ & $227.6 \pm 157.3$ & 0.202 \\
\hline HDL-C (mg/dL) & $43.9 \pm 9.7$ & $43.9 \pm 8.9$ & 0.999 \\
\hline LDL-C (mg/dL) & $107.7 \pm 25.2$ & $113.5 \pm 25.2$ & 0.500 \\
\hline CPR index & $1.8 \pm 0.7$ & $1.5 \pm 0.6$ & 0.141 \\
\hline Urinary C-peptide ( $\mu \mathrm{g} /$ day) & $85.0 \pm 36.1$ & $60.7 \pm 42.5$ & 0.083 \\
\hline Urinary albumin $(\mathrm{mg} / \mathrm{gCr})$ & $57.1 \pm 139.5$ & $89.5 \pm 137.4$ & 0.501 \\
\hline
\end{tabular}

Mean \pm SD, BMI, body mass index; HbA1c, glycated hemoglobin

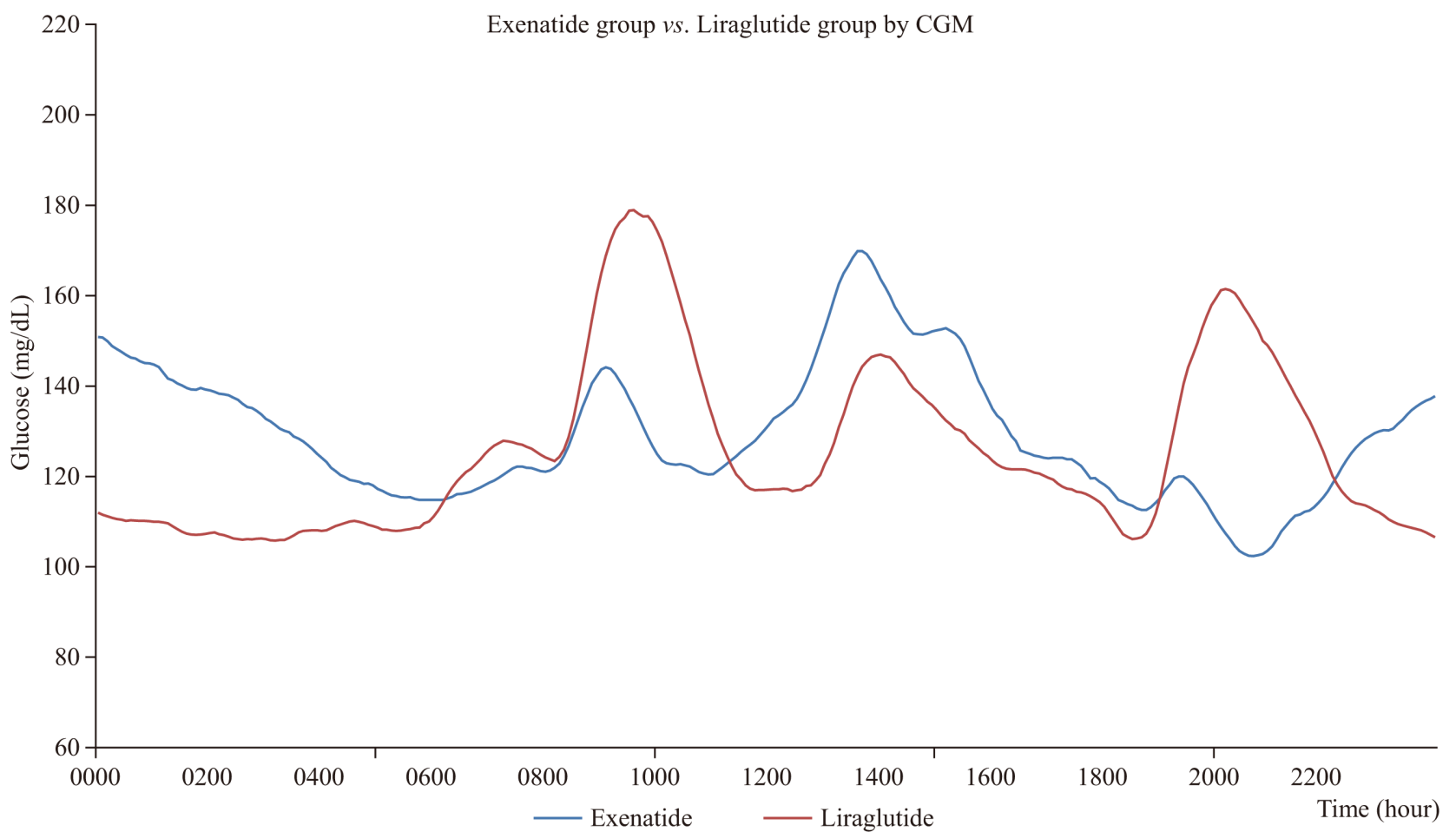

Fig. 2 The 24-hour glucose profile on day 7 of treatment with exenatide or liraglutide. 
at 3 hours after lunch (without exenatide administration) was $151.4 \pm 34.1 \mathrm{mg} / \mathrm{dL}$ in the exenatide group and $131.7 \pm 22.4$ in the liraglutide group, being lower in the latter group although the difference was not significant $(p=0.053)$. The mean blood glucose level between $0000 \mathrm{~h}$ and $0600 \mathrm{~h}$ was $126.9 \pm 27.1 \mathrm{mg} / \mathrm{dL}$ in the exenatide group and $107.1 \pm 24.0 \mathrm{mg} / \mathrm{dL}$ in the liraglutide group ( $p=0.029$ ), while the standard deviation for this period was $15.2 \pm 10.5$ and $8.7 \pm 3.8$, respectively $(p=$ 0.020 ), and both parameters were significantly lower in the liraglutide group. CGM showed no significant difference between the two groups with regard to the duration of a blood glucose level $<70 \mathrm{mg} / \mathrm{dL}$ (Table 2).

The 6-point SMBG profile data are shown in Table 3 and Fig. 3. The blood glucose level after breakfast was $112.4 \pm 36.3 \mathrm{mg} / \mathrm{dL}$ in the exenatide group and $164.2 \pm 34.7 \mathrm{mg} / \mathrm{dL}$ in the liraglutide group $(p<0.001)$, while the blood glucose level after dinner was $102.6 \pm$ $33.3 \mathrm{mg} / \mathrm{dL}$ and $172.1 \pm 40.8 \mathrm{mg} / \mathrm{dL}$, respectively $(p<$ 0.001). Blood glucose levels with significantly lower in the exenatide group at both times.

The change of blood glucose from baseline after treatment ( $\Delta$ value) was calculated to be $-33.5 \pm 53.3$ $\mathrm{mg} / \mathrm{dL}$ in the exenatide group and $-85.1 \pm 59.0 \mathrm{mg} / \mathrm{dL}$ in the liraglutide group after lunch $(p=0.012)$, while it was $-12.2 \pm 48.4 \mathrm{mg} / \mathrm{dL}$ in the exenatide group and $-60.4 \pm 44.7 \mathrm{mg} / \mathrm{dL}$ in the liraglutide group before dinner $(p=0.004)$. These changes of the glucose level after lunch and before dinner were significantly larger in the liraglutide group.

Improvement of blood glucose levels with treatment was also examined by SMBG. The improvement rate was calculated to be $-14.6 \pm 26.1 \%$ in the exenatide group and $-32.5 \pm 19.0 \%$ in the liraglutide group after lunch $(p=0.029)$, while it was $-6.8 \pm 33.7 \%$ in the exenatide group and $-32.4 \pm 19.8 \%$ in the liraglutide group before dinner $(p=0.010)$. Both improvement rates were significantly larger in the liraglutide group. However, the improvement rate after dinner was -45.6 $\pm 31.3 \%$ in the exenatide group and $-26.0 \pm 23.9 \%$ in the liraglutide group $(p=0.045)$, being significantly larger in the exenatide group. There were no significant differences of other glycemic parameters between the two groups.

\section{Discussion}

This study was the first CGM-based direct comparison of the effect of the GLP-1 receptor agonists exenatide $(10 \mu \mathrm{g} /$ day $)$ and liraglutide $(0.3-0.9 \mathrm{mg} /$ day $)$ on blood glucose levels in patients with type 2 diabetes. It demonstrated that the mean 24-hour blood glucose level, SD, and MAGE did not differ significantly between the two drugs. However, postprandial blood glucose levels were significantly lower in the exenatide group than the liraglutide group after breakfast and dinner, while the exenatide group had higher nighttime glucose levels.

GLP-1 receptor agonists are classified as short-acting or long-acting [23]. Differences in efficacy between exenatide and liraglutide have been investigated in several studies. It was reported that once-daily liraglutide treatment achieved significantly greater improvement

Table 2 Glucose fluctuation parameters derived from CGM glucose data after 7 day treatment

\begin{tabular}{lccc}
\hline & $\begin{array}{c}\text { Exenatide } \\
(\mathrm{N}=18)\end{array}$ & $\begin{array}{c}\text { Liraglutide } \\
(\mathrm{N}=17)\end{array}$ & $p$ value \\
\hline 24-h mean glucose (mg/dL) & $127.1 \pm 18.8$ & $124.7 \pm 19.4$ & 0.720 \\
SD of 24-h glucose levels (mg/dL) & $27.1 \pm 11.4$ & $26.3 \pm 10.4$ & 0.815 \\
MAGE (mg/dL) & $42.2 \pm 16.6$ & $42.2 \pm 16.2$ & 0.999 \\
\hline 0000-0600 h mean glucose (mg/dL) & $126.9 \pm 27.1$ & $107.1 \pm 24.0$ & 0.029 \\
SD of 0000-0600 h glucose levels (mg/dL) & $15.2 \pm 10.5$ & $8.7 \pm 3.8$ & 0.020 \\
After breakfast & $127.3 \pm 24.1$ & $153.4 \pm 28.7$ & 0.006 \\
0-180min mean glucose (mg/dL) & $151.4 \pm 34.1$ & $131.7 \pm 22.4$ & 0.053 \\
After lunch & $108.7 \pm 17.3$ & $141.9 \pm 24.2$ & $<0.001$ \\
0-180min mean glucose (mg/dL) & $122.4 \pm 17.8$ & $125.2 \pm 23.5$ & 0.687 \\
After dinner & $124.8 \pm 196.2$ & $83.9 \pm 90.5$ & 0.438 \\
0-180min mean glucose (mg/dL) & $6.4 \pm 15.0$ & $28.8 \pm 73.6$ & 0.215 \\
Fasting plasma glucose (mg/dL) &
\end{tabular}

Mean \pm SD, MAGE, mean amplitude of glycemic excursions; Time in hyperglycemia (>180 mg/ $\mathrm{dL})$, Time in hypoglycemia $(<70 \mathrm{mg} / \mathrm{dL})$. 
Table 3 6-point SMBG profiles. Changes in blood glucose levels pre and post each of three meals daily as a result.

\begin{tabular}{lccc}
\hline & $\begin{array}{c}\text { Exenatide } \\
(\mathrm{N}=18)\end{array}$ & $\begin{array}{c}\text { Liraglutide } \\
(\mathrm{N}=17)\end{array}$ & $p$ value \\
\hline Before breakfast & & & \\
\hline Glucose (mg/dL) & $121.6 \pm 17.8$ & $126.2 \pm 23.1$ & 0.511 \\
\hline (mg/dL) & $-29.3 \pm 28.0$ & $-41.9 \pm 29.0$ & 0.198 \\
\hline Improvement ratio (\%) & $-17.5 \pm 17.9$ & $-23.4 \pm 16.8$ & 0.315 \\
\hline After breakfast & & & \\
\hline Glucose (mg/dL) & $112.4 \pm 36.3$ & $164.2 \pm 34.7$ & $<0.001$ \\
\hline (mg/dL) & $-121.8 \pm 68.4$ & $-110.6 \pm 71.4$ & 0.634 \\
\hline Improvement ratio (\%) & $-49.5 \pm 22.3$ & $-36.3 \pm 22.1$ & 0.092 \\
\hline Before lunch & & & \\
\hline Glucose (mg/dL) & $122.9 \pm 32.0$ & $110.0 \pm 29.7$ & 0.226 \\
\hline$\Delta$ (mg/dL) & $-78.6 \pm 68.4$ & $-120.1 \pm 70.9$ & 0.087 \\
\hline Improvement ratio (\%) & $-34.3 \pm 24.1$ & $-47.9 \pm 23.0$ & 0.097 \\
\hline After lunch & & & \\
Glucose (mg/dL) & $158.7 \pm 51.2$ & $155.9 \pm 32.3$ & 0.849 \\
\hline$\Delta$ (mg/dL) & $-33.5 \pm 53.3$ & $-85.1 \pm 59.0$ & 0.012 \\
\hline Improvement ratio (\%) & $-14.6 \pm 26.1$ & $-32.5 \pm 19.0$ & 0.029 \\
\hline Before dinner & & & \\
\hline Glucose (mg/dL) & $117.6 \pm 46.8$ & $111.2 \pm 25.6$ & 0.623 \\
\hline$\Delta$ (mg/dL) & $-12.2 \pm 48.4$ & $-60.4 \pm 44.7$ & 0.004 \\
\hline Improvement ratio (\%) & $-6.8 \pm 33.7$ & $-32.4 \pm 19.8$ & 0.010 \\
\hline After dinner & $102.6 \pm 33.3$ & $172.1 \pm 40.8$ & $<0.001$ \\
\hline Glucose (mg/dL) & $-109.3 \pm 66.0$ & $-68.2 \pm 57.4$ & 0.059 \\
\hline$\Delta$ (mg/dL) & $-45.6 \pm 31.3$ & $-26.0 \pm 23.9$ & 0.045 \\
\hline Improvement ratio (\%) & & & \\
\hline Mean \pm SD, Glucose, post & & & \\
\hline
\end{tabular}

Mean \pm SD, Glucose, post-treatment blood glucose; $\Delta$, delta change (baseline - post-treatment blood glucose); Improvement ratio, $\Delta$ / baseline blood glucose.

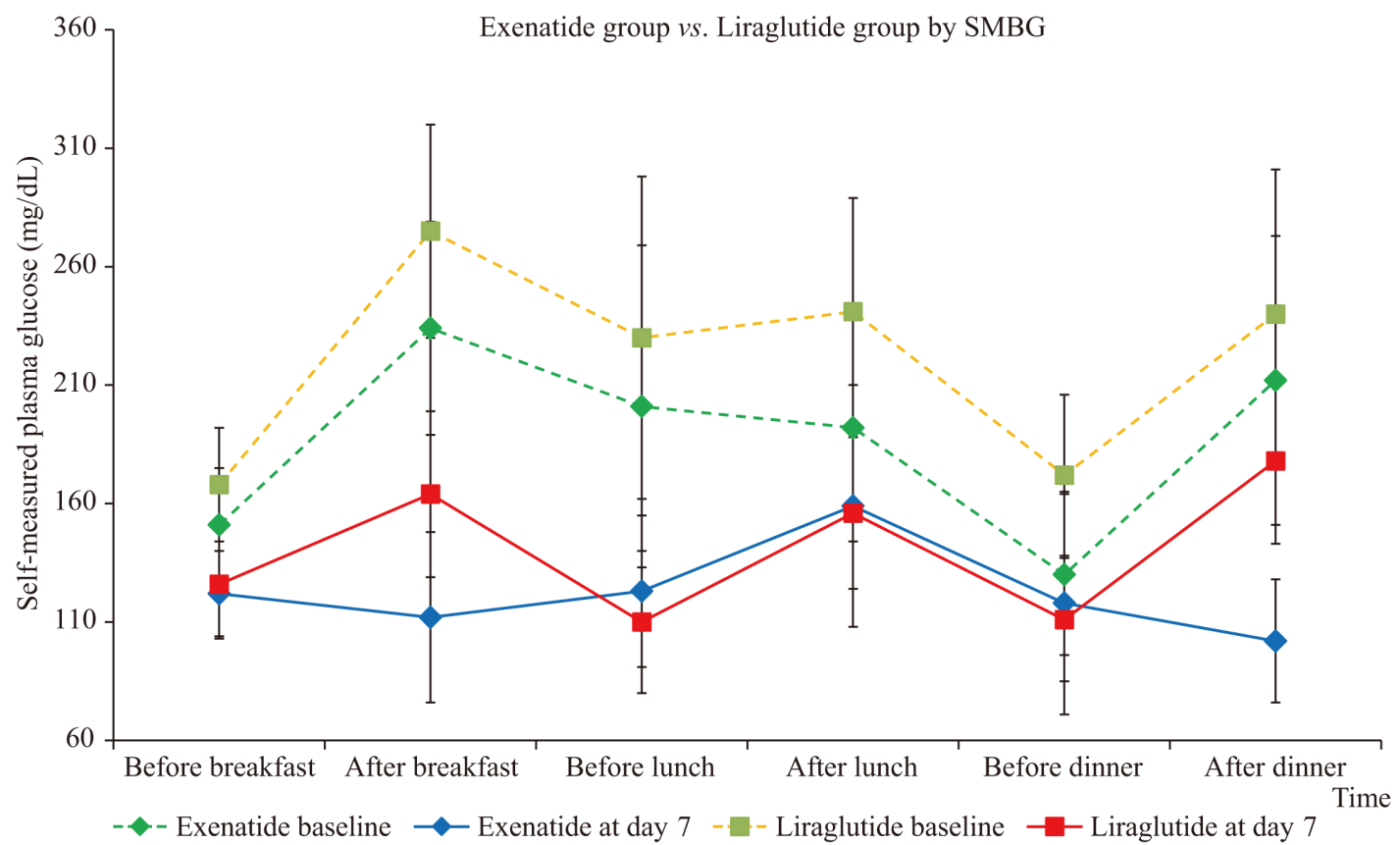

Fig. 3 The 6-point SMBG profile at baseline and on day 7 in patients treated with exenatide or liraglutide. 
of glycemic control than twice-daily exenatide treatment [24]. Liraglutide was also reported to be significantly better for patient satisfaction compared with exenatide [25]. As far as we know, there have been no previous head-to-head comparisons of these two drugs using CGM, making this the first direct comparison of 24-hour blood glucose profiles by CGM in patients with type 2 diabetes receiving treatment with exenatide or liraglutide.

One of the mechanisms through which short-acting GLP-1 receptor agonists reduce postprandial blood glucose levels is by delaying gastric emptying. However, it is known that the effect on gastric emptying decreases during continuous intravenous infusion of GLP-1 [26], suggesting that tachyphylaxis results from maintenance of a high GLP-1 concentration. Jelsing et al. assessed the effects of exenatide and liraglutide on gastric emptying, and reported that emptying was still delayed on Day 14 of exenatide treatment, whereas emptying showed less delay on Day 14 of liraglutide treatment compared with soon after the initiation of treatment [27]. In addition, Nagai et al. performed expired air analysis following treatment with liraglutide for 1 week and reported that gastric emptying was not delayed in 9 out of 16 patients [28].

Thus, gastric emptying is delayed immediately after initiating treatment with a long-acting GLP-1 receptor agonist, but persistent non-physiological high blood levels of GLP-1 lead to relatively early tachyphylaxis. Since tachyphylaxis decreases the delay of gastric emptying during treatment with long-acting GLP-1 receptor agonists, control of postprandial blood glucose may be less effective than with short-acting GLP-1 receptor agonists. In this study, we performed CGM on Day 7 of treatment and tachyphylaxis may already have occurred in the liraglutide group.

Long-acting GLP-1 receptor agonists maintain higher blood concentrations, even while fasting and at nighttime, and persistent inhibition of glucagon secretion combined with stimulation of insulin secretion may achieve a lower overall blood glucose level and lower early morning fasting glucose than those obtained with short-acting GLP-1 receptor agonists. Comparison between liraglutide and exenatide [24], or between twice-daily and once-weekly exenatide formulations [29], showed that postprandial glucose levels were markedly improved by exenatide but the preprandial decrease of glucose was small, whereas improvement of postprandial glucose was slight but the overall blood glucose level (including preprandial glucose) was reduced by liraglutide and once-weekly exenatide. In the present study, we also found good overall blood glucose control throughout the day in the liraglutide group, with nocturnal glucose levels being lower than in the exenatide group. However, in contrast to the above reports, we found no difference of early morning fasting blood glucose levels between the two groups, possibly because our subjects were receiving concomitant treatment with sulfonylureas.

There were no significant differences of the 24-hour mean glucose level, SD, and MAGE in the present study, possibly because the times when strong and weak hypoglycemic effects are observed differ considerably between exenatide and liraglutide (Fig. 2), but such differences are masked by calculation of the 24-hour mean glucose level, SD, and MAGE.

The CGM data obtained in this study indicate that it is difficult to explain differences of the hypoglycemic effect between drugs with different durations of action in terms of 24-hour glucose parameters, and that changes of blood glucose need to be assessed over a shorter period. It is especially difficult to assess changes of nocturnal glucose levels by SMBG and CGM is very useful in this respect.

In the present study, sulfonylureas were administered concomitantly in both groups. The effects of combined use of GLP-1 receptor agonists and drugs other than sulfonylureas have been assessed by CGM. In this study, GLP-1 receptor agonists were not combined with metformin, which is a first-line drug in the ADA/EASD consensus algorithm, because this combination was not approved in Japan when the study was conducted. Exenatide improves postprandial blood glucose, but has a short duration of action that allows nocturnal glucose levels to increase. When exenatide is used in combination with a biguanide or basal insulin to control nocturnal glucose levels and compensate for its short duration of action, the result is smaller glucose excursions and good glycemic control. Liraglutide is a long-acting drug that can reduce the overall blood glucose level both before and after meals, but it has a smaller effect on postprandial blood glucose than exenatide in Japanese patients. This suggests that drugs to reduce postprandial blood glucose, such as $\alpha$-glucosidase inhibitors or glinides, should be used with liraglutide, or it could be combined with ultrarapid-acting and rapid-acting insulins that improve postprandial blood glucose. Furthermore, use of lira- 
glutide in combination with basal-bolus insulin could allow the total insulin dose to be decreased and also reduce blood glucose fluctuations.

The first limitation of this study was the small number of subjects $(n=40)$. No significant differences were observed between the two treatment groups at baseline, but $\mathrm{HbA1c}$, the duration of diabetes, FPG, and SMBG data were slightly higher in the liralutide group. Accordingly, is possible that differences of these baseline values might have had an influence on the results.

In addition, the patients were hospitalized and CGM was performed from 7 days after the initiation of GLP-1 receptor agonists treatment, so changes of blood glucose were assessed a short time after starting treatment. Since we were not able to compare the changes of blood glucose between before and after initiation of treatment by CGM, we used the 6-point SMBG profile instead. Future studies should have a larger sample size and include outpatients. Also, long-term assessment of the effect of short-acting GLP-1 receptor agonists on gastric emptying has not been performed, although long-term use of these drugs may cause tachyphylaxis, indicating the need to confirm their influence on gastric emptying effect following a longer treatment period.

In conclusion, there were no significant differences of glucose fluctuations between patients treated with exenatide or liraglutide, but the 24-hour blood glucose profile was different. Each of these agents has certain advantages and disadvantages, suggesting that we should select GLP-1 receptor agonist therapy according to the blood glucose profile of the patient.

\section{Conflict of Interest Statement}

We thank all the physicians who participated in this study.

Yasuo Terauchi received honoraria for lectures from MSD K.K.; Ono Pharmaceutical Co., Ltd.; Nippon Boehringer Ingelheim Co., Ltd.; Novartis Pharma K.K.; Takeda Pharmaceutical Co., Ltd.; Mitsubishi Tanabe Pharma Corp.; Daiichi Sankyo Co., Ltd.; Sanwa Kagaku Kenkyusho Co., Ltd.; Kowa Pharmaceutical Co., Ltd.; Novo Nordisk Pharma Ltd.; Eli Lilly Japan K.K.; Sanofi K.K.; Shionogi \& Co., Ltd.; Bayer Yakuhin, Ltd.; and AstraZeneca K.K. and obtained research support from MSD K.K.; Ono Pharmaceutical Co., Ltd.; Nippon Boehringer Ingelheim Co., Ltd.; Novartis Pharma K.K.; Takeda Pharmaceutical Co., Ltd.; Mitsubishi Tanabe Pharma Corp.; Daiichi Sankyo Co., Ltd.; Sanwa Kagaku Kenkyusho Co., Ltd.; Novo Nordisk Pharma Ltd.; Eli Lilly Japan K.K.; Sanofi K.K.; Dainippon Sumitomo Pharma Co., Ltd.; Shionogi \& Co., Ltd.; Bayer Yakuhin, Ltd.; Astellas Pharma, Inc.; Pfizer Japan, Inc.; and AstraZeneca K.K.

Tadashi Yamakawa received honoraria for lectures from MSD K.K.; Kowa Pharmaceutical Co., Ltd.; Novo Nordisk Pharma Ltd.; Sanofi K.K. and obtained research support from AstraZeneca K.K.

Jo Nagakura, Masataka Taguri, Hirohisa Tsuchiya, Erina Shigematsu, Jun Suzuki, Satoshi Morita, Kazuaki Kadonosono declare that they have no conflict of interest.

\section{Acknowledgements}

This work was conducted independently; no company or institution supported it financially. We appreciate Mrs. Yamagiwa and Seki for secretarial assistance.

\section{References}

1. Monnier L, Mas E, Ginet C, Michel F, Villon L, et al. (2006) Activation of oxidative stress by acute glucose fluctuations compared with sustained chronic hyperglycemia in patients with type 2 diabetes. JAMA 295: 1681-1687.

2. Brunner EJ, Shipley MJ, Witte DR, Fuller JH, Marmot MG (2006) Relation between blood glucose and coronary mortality over 33 years in the Whitehall Study. Diabetes Care 29: 26-31.

3. Esposito K, Giugliano D, Nappo F, Marfella R (2004) Regression of carotid atherosclerosis by control of post- prandial hyperglycemia in type 2 diabetes mellitus. Circulation 110: 214-219.

4. Muggeo M, Zoppini G, Bonora E, Brun E, Bonadonna RC, et al. (2000) Fasting plasma glucose variability predicts 10-year survival of type 2 diabetic patients: the Verona Diabetes Study. Diabetes Care 23: 45-50.

5. Meigs JB, Nathan DM, D'Agostino RB, Wilson PW (2002) Fasting and postchallenge glycemia and cardiovascular disease risk: the Framingham Offspring Study. Diabetes Care 25: 1845-1850.

6. Takei Y, Tomiyama H, Tanaka N, Yamashina A (2007) 
Close relationship between sympathetic activation and coronary microvascular dysfunction during acute hyperglycemia in subjects with atherosclerotic risk factors. Circ J 71: 202-206.

7. Chiasson JL, Josse RG, Gomis R, Hnanefeld M, Karasik A, et al. (2003) Acarbose treatment and the risk of cardiovascular disease and hypertension in patients with impaired glucose tolerance: the STOP-NIDDM trial. JAMA 290: 486-494.

8. Kovatchev BP, Otto E, Cox D, Gonder-Frederick L, Clarke W (2006) Evaluation of a new measure of blood glucose variability in diabetes. Diabetes Care 29: 2433-2438.

9. Drucker DJ, Nauck MA (2006) The incretin system: glucagon-like peptide-1 receptor agonists and dipeptidyl peptidase- 4 inhibitors in type 2 diabetes. Lancet 368: 1696-1705.

10. Eng J, Kleinman WA, Singh L, Singh G, Raufman JP (1992) Isolation and characterization of exendin-4, an exendin-3 analogue, from Heloderma suspectum venom. Further evidence for an exendin receptor on dispersed acini from guinea pig pancreas. $J$ Biol Chem 267: 7402-7405.

11. Kolterman OG, Kim DD, Shen L, Ruggles JA, Nielsen LL, et al. (2005) Pharmacokinetics, pharmacodynamics, and safety of exenatide in patients with type 2 diabetes mellitus. Am J Health Syst Pharm 62: 173-181.

12. Cvetkovic RS, Plosker GL (2007) Exenatide a review of its use in patients with type 2 diabetes mellitus (as an adjunct to metformin and/or a sulfonylurea). Drugs 67: 935-954.

13. Heine RJ, Van Gaal LF, Johns D, Mihm MJ, Widel $\mathrm{MH}$, et al. (2005) Exenatide versus insulin glargine in patients with suboptimally controlled type 2 diabetes: a randomized trial. Ann Intern Med 143: 559-569.

14. Degn KB, Brock B, Juhl CB, Djurhuus CB, Grubert J, et al. (2004) Effect of intravenous infusion of exenatide (synthetic exendin-4) on glucose-dependent insulin secretion and counterregulation during hypoglycemia. Diabetes 53: 2397-2403.

15. Edwards CM, Stanley SA, Davis R, Brynes AE, Frost GS, et al. (2001) Exendin-4 reduces fasting and postprandial glucose and decreases energy intake in healthy volunteers. Am J Physiol Endocrinol Metab 281: E155-E161.

16. Egan JM, Meneilly GS, Habener JF, Elahi D (2002) Glucagon-like peptide-1 augments insulin-mediated glucose uptake in the obese state. J Clin Endocrinol Metab 87: 3768-3773.

17. Kolterman OG, Buse JB, Fineman MS, Gaines E, Heintz S, et al. (2003) Synthetic exendin-4 (exenatide) significantly reduces postprandial and fasting plasma glucose in subjects with type 2 diabetes. J Clin Endocrinol Metab 88: 3082-3089.
18. Poon T, Nelson P, Shen L, Mihm M, Taylor K, et al. (2005) Exenatide improves glycemic control and reduces body weight in subjects with type 2 diabetes: a dose-ranging study. Diabetes Technol Ther 7: 467-477.

19. Agerso H, Vicini P (2003) Pharmacodynamics of NN2211, a novel long acting GLP-1 derivative. Eur $J$ Pharm Sci 19: 141-150.

20. Bolinder J, Hagstrom-Toft E, Ungerstedt U, Arner P (1997) Self-monitoring of blood glucose in type I diabetic patients: comparison with continuous microdialysis measurements of glucose in subcutaneous adipose tissue during ordinary life conditions. Diabetes Care 20: 64-70.

21. Irace C, Fiorentino R, Carallo C, Scavelli F, Gnasso A (2011) Exenatide improves glycemic variability assessed by continuous glucose monitoring in subjects with type 2 diabetes. Diabetes Technol Ther 13: 1261-1263.

22. Mori Y, Taniguchi Y, Sezaki K, Yokoyama J, Utsunomiya K (2011) Liraglutide Narrows the Range of Circadian Glycemic Variations in Japanese Type 2 Diabetes Patients and Nearly Flattens These Variations in Drug-Naive Type 2 Diabetes Patients: A Continuous Glucose Monitoring-Based Study. Diabetes Technol Ther 13: 1139-1144.

23. Meier JJ (2012) GLP-1 receptor agonists for individualized treatment of type 2 diabetes mellitus. Nat Rev Endocrinol 8: 728-742.

24. Buse JB, Rosenstock J, Sesti G, Schmidt WE, Montanya E, et al. (2009) Liraglutide once a day versus exenatide twice a day for type 2 diabetes: a 26-week randomised, parallel-group, multinational, open-label trial (LEAD6). Lancet 374: 39-47.

25. Schmidt WE, Christiansen JS, Hammer M, Zychma MJ, Buse JB (2011) Patient-reported outcomes are superior in patients with Type 2 diabetes treated with liraglutide as compared with exenatide, when added to metformin, sulphonylurea or both: results from a randomized, open-label study. Diabet Med 28: 715-723.

26. Nauck MA, Kemmeries G, Holst JJ, Meier JJ (2011) Rapid tachyphylaxis of the glucagon-like peptide 1 -induced deceleration of gastric emptying in humans. Diabetes 60: 1561-1565.

27. Jelsing J, Vrang N, Hansen G, Raun K, Tang-Christensen M, et al. (2012) Liraglutide: short-lived effect on gastric emptying -- long lasting effects on body weight. Diabetes Obes Metab 14: 531-538.

28. Nagai Y, Hashimoto E, Oikawa R, Asai S, Terashima Y, et al. (2014) Differing effects of liraglutide on gastric emptying in Japanese patients with type 2 diabetes. Diabetes Obes Metab 16: 573-576.

29. Ji L, Onishi Y, Ahn CW, Agarwal P, Chou CW, et al. (2013) Efficacy and safety of exenatide once-weekly vs exenatide twice-daily in Asian patients with type 2 diabetes mellitus. J Diabetes Investig 4: 53-61. 